\title{
OPEN Roles for the RNA polymerase III regulator MAFR-1 in regulating sperm quality in Caenorhabditis elegans
}

\begin{abstract}
Amy M. Hammerquist ${ }^{1,2}$ \& Sean P. Curran ${ }^{1,2,3 凶}$
The negative regulator of RNA polymerase (pol) III mafr-1 has been shown to affect RNA pol III transcript abundance, lipid biosynthesis and storage, progeny output, and lifespan. We deleted mafr1 from the Caenorhabditis elegans genome and found that animals lacking mafr-1 replicated many phenotypes from previous RNAi-based studies and discovered a new sperm-specific role. Utilizing a yeast two-hybrid assay, we discovered several novel interactors of MAFR-1 that are expressed in a sperm- and germline-enriched manner. In support of a role for MAFR-1 in the male germline, we found mafr-1 null males have smaller spermatids that are less capable in competition for fertilization; a phenotype that was dependent on RNA pol III activity. Restoration of MAFR-1 expression specifically in the germline rescued the spermatid-related phenotypes, suggesting a cell autonomous role for MAFR-1 in nematode male fertility. Based on the high degree of conservation of Maf1 activity across species, our study may inform similar roles for Maf1 and RNA pol III in mammalian male fertility.
\end{abstract}

Canonically characterized as a negative regulator of RNA polymerase (pol) III, MAF1 was originally discovered and has been extensively studied in Saccharomyces cerevisiae ${ }^{1-3}$. Since its discovery, MAF1 has been identified across diverse eukaryotic clades ${ }^{2-7}$. Perturbation of MAF1 activity leads to, in addition to increased RNA pol III activity, an increase in intracellular lipid abundance ${ }^{8-10}$ and in some instances altered lifespan ${ }^{11,12}$. The majority of studies of MAF1 have been conducted in single-cell systems (as S. cerevisiae) or cultured mammalian cells ${ }^{4,10,13,14}$, but relatively few studies have probed the function of $M A F 1$ within the context of a complete animal $^{8,15-17}$. In Caenorhabditis elegans, RNA interference (RNAi) knockdown of MAF1 homolog mafr-1 results in increased RNA pol III activity, increased intestinal lipid accumulation (as well as increased expression of lipid biogenesis genes), and increased expression of the vitellogenin family of lipid transport proteins ${ }^{8}$. As vitellogenesis is the process by which lipids are transferred to developing oocytes, these findings imply a role for MAFR-1 in reproduction, but this has not yet been fully investigated.

Although RNA pol III activity has, to our knowledge, not been directly implicated in fertility, many processes affected by RNA pol III activity do affect reproductive output. Drosophila with disrupted ribosome biogenesis display a Minute phenotype characterized by delayed development, short and thin bristles, and impaired fertility and viability ${ }^{18}$. Furthermore, mice lacking $Z f n 384$, a protein whose sub-cellular location was highly correlated with that of RNA pol III in human embryonic stem cells, have fertility defects and impaired spermatogenesis ${ }^{19,20}$. While mature sperm are widely accepted to be transcriptionally and translationally quiescent ${ }^{21,22}$, as a repressor of RNA pol III activity, MAFR-1 may still be acting in developing germ cells to prevent erroneous RNA pol III transcription.

In all sexually reproducing species, sperm are produced in great excess of oocytes, and must compete with each other to successfully fertilize an oocyte. Generally, larger sperm are able to travel faster than smaller sperm, and therefore have a competitive advantage ${ }^{23-25}$. As a hermaphroditic species, C. elegans presents a unique situation: the sperm produced by hermaphrodites are capable of fertilizing oocytes but are almost completely outcompeted by male sperm, when mated $^{26}$. In this work, we explore the role of MAFR-1 in maintaining male sperm quality in C. elegans, which affects sperm quality metrics such as size and activation capacity, as well as competitive advantage over hermaphrodite sperm.

${ }^{1}$ Leonard Davis School of Gerontology, University of Southern California, Los Angeles, CA, USA. ${ }^{2}$ Department of Molecular and Computational Biology, Dornsife College of Letters, Arts, and Science, University of Southern California, Los Angeles, CA, USA. ${ }^{3}$ Norris Comprehensive Cancer Center, University of Southern California, LoS Angeles, CA, USA. ${ }^{\boxplus}$ email: spcurran@usc.edu 


\begin{abstract}
Results
Characterization of a mafr-1 null mutant. Previous studies of mafr-1 in C. elegans have utilized RNAi-based approaches ${ }^{8}$ and a gain-of-function allele of $m a f r-1{ }^{16}$ leaving the true loss-of-function phenotype unknown. To better examine the biological functions of MAFR-1, we assessed the impact of a true molecular null allele of mafr-1, a CRISPR-generated and sequence confirmed deletion of the entire mafr-1/C43H8.2 coding sequence from start to stop, hereafter referred to as mafr-1 (KO) (Fig. 1a). Surprisingly, a total loss of mafr-1 did not result in changes in developmental timing (Fig. 1b) or overall organismal health, as mafr-1 (KO) animals had similar lifespans to wild type (WT) controls (Fig. 1c, Fig. S1a). While mafr-1 expression was undetectable in mafr-1 (KO) animals (Fig. 1d), expression of genes downstream in the CEOP1628 operon, arch-1 and B0511.6, were unaffected (Fig S1b). Consistent with its canonical role as a negative regulator of RNA pol III activity and previously observed phenotypes for mafr-1 $\mathrm{RNAi}^{2,3,8}$, mafr-1 (KO) animals showed increased expression of RNA pol III transcripts, including three tRNAs: initiator Methionine, Tryptophan, and Iso-leucine (Fig. 1d). Similarly, mafr-1 (KO) animals displayed increased intracellular lipid abundance relative to age-matched WT control animals (Fig. 1e), as expected from previous studies ${ }^{8-10,16}$. Additionally, the expression of vitellogenins, which deliver lipids from the intestine to the germline to drive reproduction, was previously demonstrated to increase in mafr-1 RNAi treated animals and diminished by mafr-1 overexpression ${ }^{8}$, and was increased mafr-1 (KO) animals relative to WT controls at the transcriptional (Fig. 1f), but not the protein, level (Fig. S1c). Our previous investigations revealed that overexpression of mafr-1 can influence reproductive output ${ }^{8}$. Although the total reproductive output between mafr-1 (KO) animals and WT controls were not significantly different (Fig. 1g), peak reproductive output appeared delayed in mafr-1 (KO) animals (Fig. 1h). Taken together, these results indicate that mafr-1 (KO) animals share several of the previously documented mafr-1-associated phenotypes, without compromised overall health.
\end{abstract}

MAFR-1 interacts with sperm-enriched proteins. MAF1 has been shown to physically interact with components of the RNA pol III complex ${ }^{27-30}$, but other direct interactors remain elusive. In order to look for novel protein interactors with MAFR-1, we performed a yeast two-hybrid screen using MAFR-1 as bait. We identified 62 putative protein-protein interactors of MAFR-1 (Table S1), which represent GO-terms comprising multiple essential cellular processes (Fig. S2a). Among these hits, we defined a novel class of germline- or spermatid-enriched ${ }^{31}$ putative MAFR-1 interactors: SSS-1, F48C1.6, and MSP-53. Because a role for MAF1 in germ cells has not been previously described, we chose these putative interactors for further analysis. We first examined the RNA expression levels of each putative interactor in wild type and mafr-1 (KO) animals by qPCR, which revealed increased expression of $s s s-1$ and $m s p-53$, but not F48C1.6 relative to WT (Fig. S1b). Next, we confirmed the physical interaction of MAFR-1 with SSS-1 (Fig. 2a), and F48C1.6 (Fig. 2b) biochemically by coexpression in E.coli followed by affinity purification MAFR-1, which facilitated the co-purification of each interactor (Fig. 2a,b). While we observed clear enrichment for SSS-1 and F48C1.6, our ability to measure enrichment of MSP-53 was confounded by its association with the Ni-NTA resin (Fig. S2c); a quality likely associated with its known capacity to mediate multiple interactions ${ }^{32,33}$. Based on their enriched expression in spermatids, we next assessed the impact of reducing the expression of SSS-1 or F48C1.6 by RNAi on sperm quality. Sperm size is a well-established quality that influence male reproductive success through sperm competition ${ }^{24}$. Caenorhabditis elegans males produce sperm that are significantly larger than hermaphrodite sperm and this size difference facilitates male competitive advantage when mating occurs. As expected based on their enrichment in germ cells, RNAi of sss- 1 and $m s p$-53, but not F48C1.6, resulted in decreased spermatid size (Fig. 2c,d, Fig. S2d-e).

In addition to size, the speed of male sperm is greater than hermaphrodite-derived sperm, which also enhances male competitive advantage for fertilizing an oocyte ${ }^{24}$. To become motile, inactive spermatids must become activated by developing pseudopodia, a sophisticated process under the control of genetic and environmental factors ${ }^{32,34,35}$. In the laboratory, isolated spermatids can be activated in vitro by exposure to Pronase $\mathrm{s}^{34,35}$. RNAi of these potential interactors resulted in no significant change in activation (Fig. S2f); however, as several validated Spe genes are not sterile when inactivated by $\mathrm{RNAi}^{36,37}$, future studies of the role of MAFR-1 and sperm-enriched proteins is warranted. Nevertheless, in light of these findings, we hypothesized that MAFR-1 might also function in the male germline to influence reproductive success.

MAFR-1 regulates male sperm fitness. To investigate the role of MAFR-1 in spermatogenesis, we looked at spermatid size and activation. We found that mafr-1 $(\mathrm{KO})$ males produced significantly smaller spermatids (Fig. 3a, Fig. S3a). Interestingly, while some physiological responses, including reproduction, are sensitive to diet type ${ }^{8,16,35,38-43}$, the reduced size phenotype in mafr-1 (KO) males is also observed on the HT115 E.coli K-12 bacterial diet (Fig. S3b-c). Next, we sought to determine the causal relationship between MAFR-1 negative regulation of RNA pol III activity and spermatid size. BRF-1 is a transcription factor required for proper RNA pol III activity ${ }^{4-48}$. Because $m a f r-1$ (KO) animals have increased RNA pol III activity, we used RNAi against $b r f-1$ and found that male spermatid size was restored in mafr-1 (KO) animals (Fig. 3b, Fig. S3d). These results suggest a role for MAFR-1 in regulating sperm quality and competitive ability.

We also found that mafr-1 (KO) male sperm are less capable of activation upon treatment with Pronase (Fig. 3c). Thus, mafr-1 (KO) males have smaller sperm with a reduced capacity to mature, which could impact their ability to fertilize hermaphrodite oocytes. Interestingly, we found that RNAi knockdown of brf-1 did not significantly affect spermatid activation in WT or mafr-1 (KO) animals (Fig. 3d).

In order to test the physiological consequence of mafr-1 (KO) on sperm fitness phenotypes, we designed an assay to assess sperm quality ${ }^{35}$. When hermaphrodites are mated to WT males, nearly all resulting progeny are derived from male sperm ${ }^{26}$. This can be visualized if males harboring a wrmScarlet transgene are used for mating; progeny derived from male sperm express wrmScarlet while progeny stemming from hermaphrodite self-sperm 
a

\section{LG I}

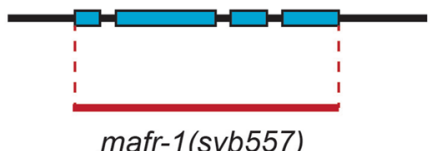

mafr-1(syb557)

C

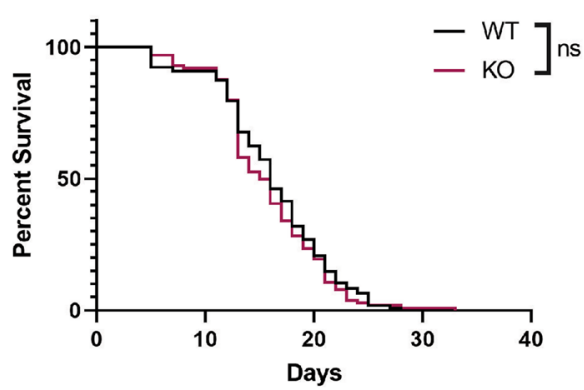

e

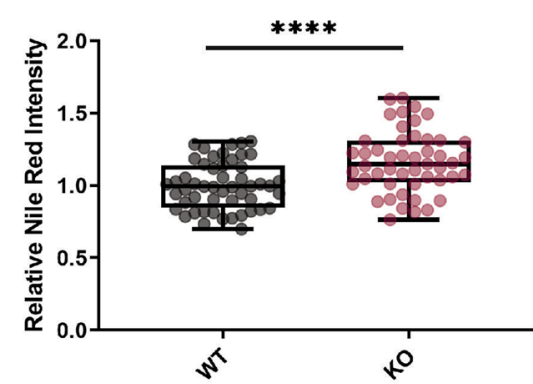

g

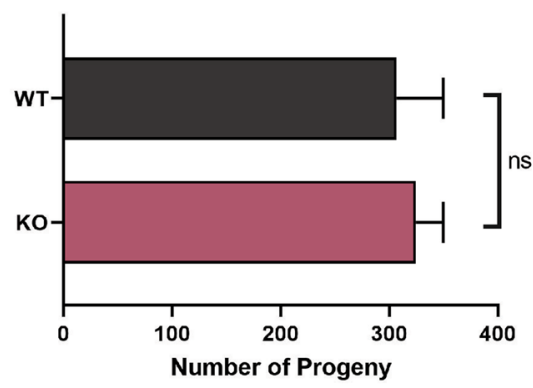

$\mathrm{b}$

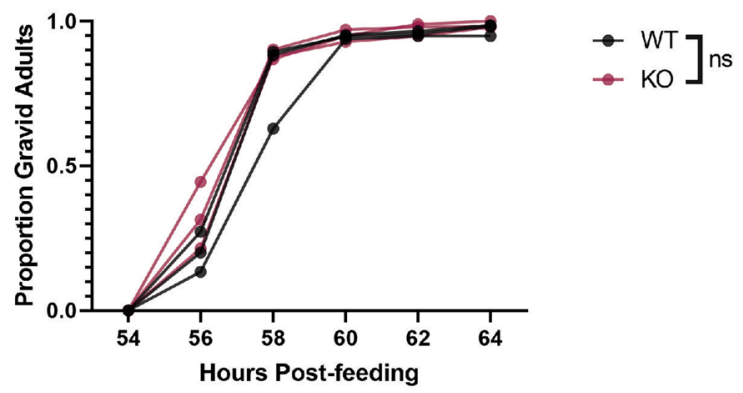

d

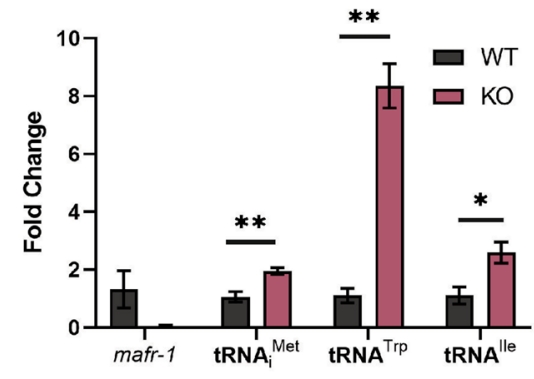

f

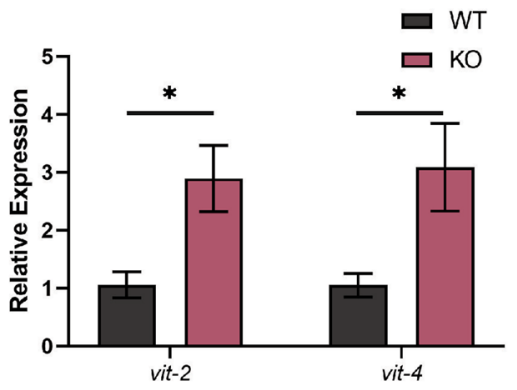

$\mathrm{h}$

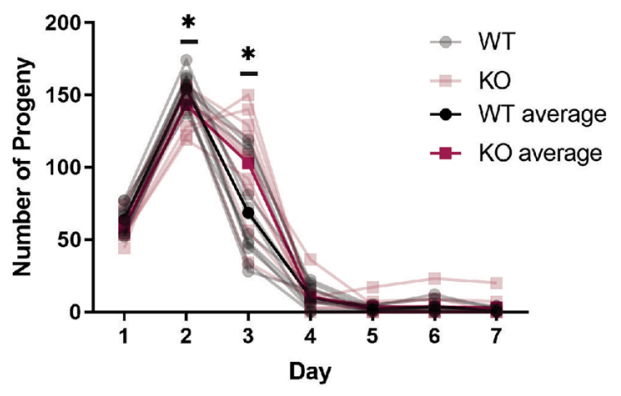

Figure 1. mafr-1 (KO) produces fertile, viable hermaphrodites. (a) Schematic diagram showing region deleted in mafr-1(syb557), a CRISPR-generated and sequence confirmed deletion of the entire mafr-1/C43H8.2 coding sequence from the start to stop codons, hereafter referred to as mafr-1 (KO) (Adobe Illustrator v24.3). (b) Developmental time course of WT and mafr-1 (KO) hermaphrodites. Each line represents one population of $>100$ animals. (c) Lifespan survival assay of WT and mafr-1 (KO) hermaphrodites. Individual lines represent average of two or three biological replicates of 50-worm populations (see Fig. S1a). No significant difference was found by Log-rank (Mantel-Cox) test. (d) Quantitative PCR expression analysis of mafr-1 and three tRNA RNA pol III transcripts: initiator Methionine, Tryptophan, and Iso-leucine. (e) Lipid abundance as measured by Nile Red staining intensity. (f) Quantitative PCR expression of vit-2 and vit-4. (g) Total brood size of WT and mafr-1 (KO) hermaphrodites $(\mathrm{n}>10)$. (h) Time course showing reproductive output of WT and mafr-1 $(\mathrm{KO})$ hermaphrodites throughout reproductive span. Unless otherwise specified, statistical comparisons made by Student's t-test (two-tailed). ns $=$ no significance, ${ }^{*} \mathrm{p}<0.05,{ }^{* *} \mathrm{p}<0.01,{ }^{* *} \mathrm{p}<0.001,{ }^{* * *} \mathrm{p}<0.0001$. 
a

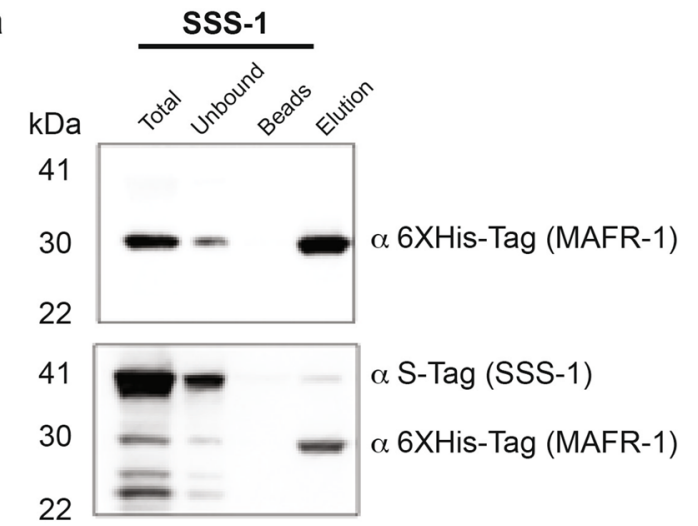

b

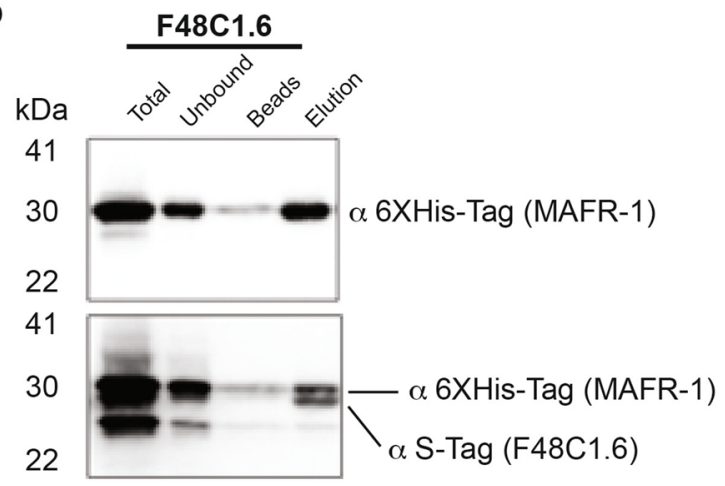

C

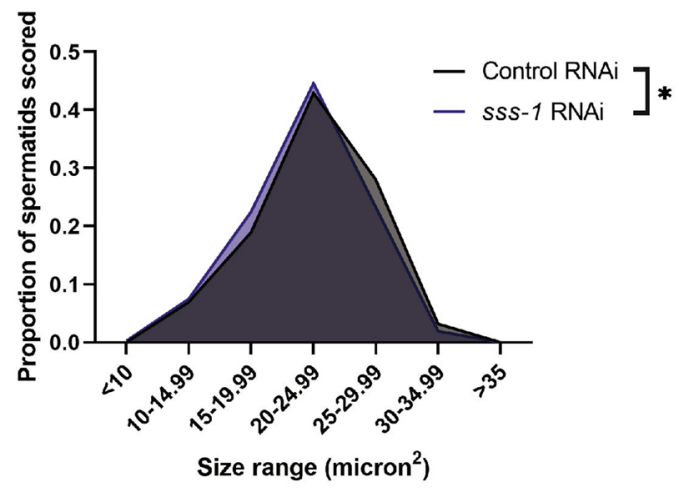

d

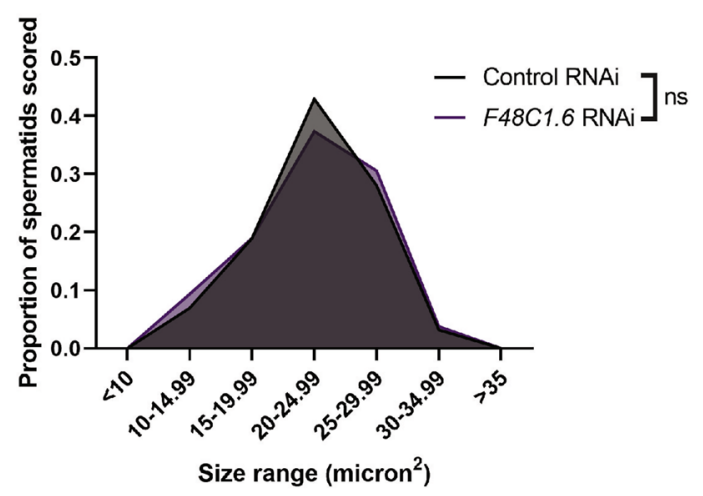

Figure 2. MAFR-1 interacts with SSS-1 and F48C1.6, which affect spermatid size. (a,b) Western Blot analysis of Ni-NTA column co-purification of MAFR-1 and SSS-1 (a), and MAFR-1 and F48C1.6 (b). (c,d) Spermatid size in day 1 adult males following RNAi depletion of sss-1 (c), and F48C1.6 (d). See Supplemental Data Set for further statistical analysis. The same Control RNAi data are depicted in (c,d). Experiments done in biological triplicate, statistical comparisons made by Student's t-test (two-tailed). ns $=$ no significance, ${ }^{*} \mathrm{p}<0.05,{ }^{* *} \mathrm{p}<0.01$, ${ }^{* * *} \mathrm{p}<0.001,{ }^{* * * *} \mathrm{p}<0.0001$.

do not express wrmScarlet (Fig. 3e). Thus, the proportion of non-Red progeny corresponds to utilization of self-sperm. When WT males expressing a CRISPR-integrated, single-copy, ubiquitously-expressed wrmScarlet marker were mated to WT hermaphrodites, nearly all subsequent progeny over the reproductive span expressed wrmScarlet, and were therefore derived from male sperm acquired through mating (Fig. $3 \mathrm{f}$ ). In contrast, when wrmScarlet-expressing mafr-1 $(\mathrm{KO})$ males were mated to WT hermaphrodites, 10 of 28 individuals produced progeny that were non-Red (as opposed to 1 of 28 in WT males), which indicates a significant proportion of self-sperm-fertilized progeny, and implies impairment of these mafr-1 (KO) male sperm to outcompete WT hermaphrodite self-sperm. Taken together, these data reveal a novel role for MAFR-1 in spermiogenesis.

MAFR-1 impacts sperm quality cell autonomously. One possible explanation for the discrepancy of phenotypes resulting from RNAi and genetic studies, is the lack of uniformity of RNAi across different tissues $^{49-53}$. To assess whether MAFR-1 functions cell autonomously in the germline to regulate sperm quality, we restored MAFR-1 in the germline of mafr-1 (KO) animals, driving mafr-1 expression with the pie-1 promoter $^{54-56}$ (Fig. S4a). Animals with germline-specific rescue of mafr-1 developed normally into fertile adults, but exhibited a slight developmental delay (Fig. S4b). Germline-specific expression of mafr-1 in mafr-1 (KO) males restored spermatid size to that of WT males (Fig. 4a, Fig. S4c), while partially restoring spermatid activation (Fig. 4b). Importantly, germline rescue of MAFR-1 also restored the competitive ability of mafr-1(KO) male sperm in our mating assay, with only 2 of 25 Rescue-mated WT hermaphrodites producing non-Red progeny (Fig. 4c), without affecting total brood size (Fig. S4d). These data suggest a cell-autonomous role for MAFR-1 in the male germline and collectively our study reveals a role for MAFR-1 in multiple parameters of sperm quality and male reproductive fitness (Fig. $4 \mathrm{~d}$ ).

\section{Discussion}

In light of the conflicting phenotypes associated with the loss of MAF1 by RNA interference (RNAi), genetic mutations in metazoans ${ }^{8,15-17,43}$, and in cultured cell models ${ }^{10}$, we characterized a loss-of-function allele in a second metazoan model. mafr-1 (KO) C. elegans display no gross defects- males have no obvious structural changes to the male copulatory organ when compared to WT (Fig. S3e-f) and are generally healthy (Fig. 1b,c), 
a

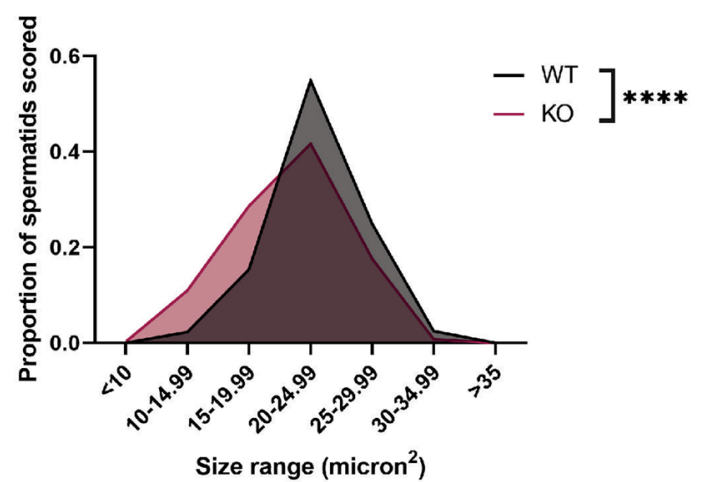

C

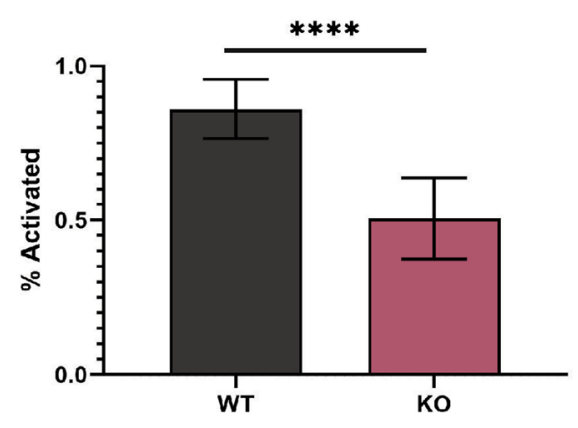

e

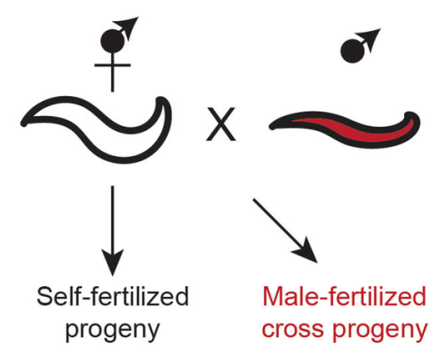

b

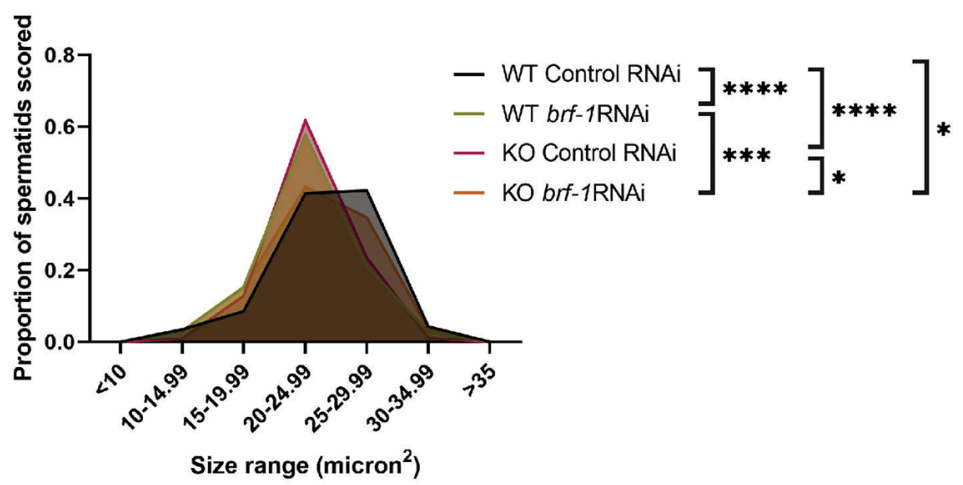

d

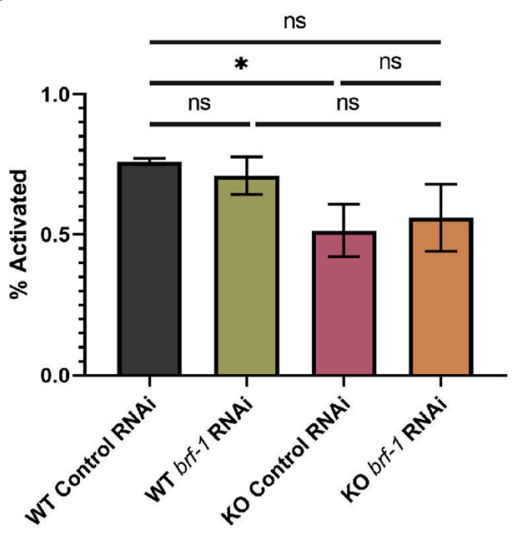

f

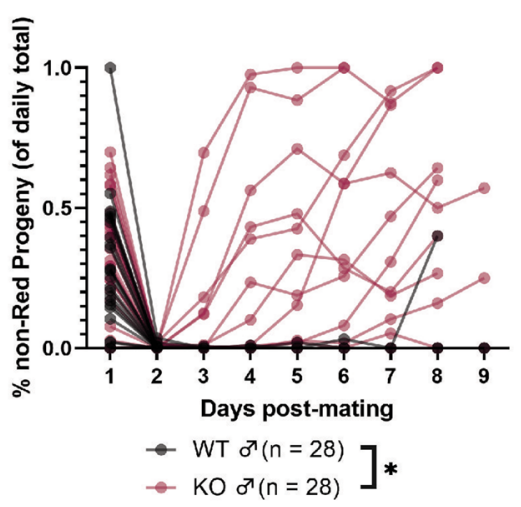

Figure 3. mafr-1 (KO) males have diminished sperm quality. (a) Spermatid size in WT and mafr-1 (KO) males. See Supplemental Data Set for further statistical analysis. (b) Spermatid size of day 2 adult WT and mafr-1 (KO) males following RNAi knockdown of brf-1. See Supplemental Data Set for further statistical analysis. (c) In vitro Pronase activation of WT and mafr-1 (KO) male spermatids. (d) In vitro Pronase activation of day 2 adult WT and mafr-1 (KO) males following RNAi knockdown of brf-1. (e) Schematic diagram showing setup of male sperm competition mating assay (Adobe Illustrator v24.3). (f) Proportion of progeny fertilized by selfsperm from WT- and mafr-1 (KO)-mated hermaphrodites. Experiments done in biological triplicate. Statistical comparisons made by Student's t-test (two-tailed). ns $=$ no significance, ${ }^{*} \mathrm{p}<0.05,{ }^{* *} \mathrm{p}<0.01,{ }^{* * *} \mathrm{p}<0.001$, ${ }^{* * * *} \mathrm{p}<0.0001$.

with brood sizes comparable to WT (Fig. 1g). Nevertheless, the novel sperm-enriched interactors discovered in a yeast two-hybrid screen (Fig. 2, Fig. S2) prompted us to investigate the roles of MAFR-1 in the germline, specifically of males, which had not been previously studied. Recent work has found MAF1 expression in embryonic stem cells drives differentiation ${ }^{13}$. Caenorhabditis elegans possess only one population of stem cells-the germline-which originate from a single cell and become progressively more mature as they migrate through the gonad away from the distal tip cell ${ }^{57}$. In fact, previous studies have found mafr-1 enriched in the C. elegans germline, but the significance of this finding has never been studied ${ }^{58-60}$.

While previous work documented altered vitellogenin expression at both the transcriptional and protein level in response to mafr-1 $\mathrm{RNAi}^{8}$, we observed altered vitellogenin expression only at the mRNA level (Fig. 1f, 
a

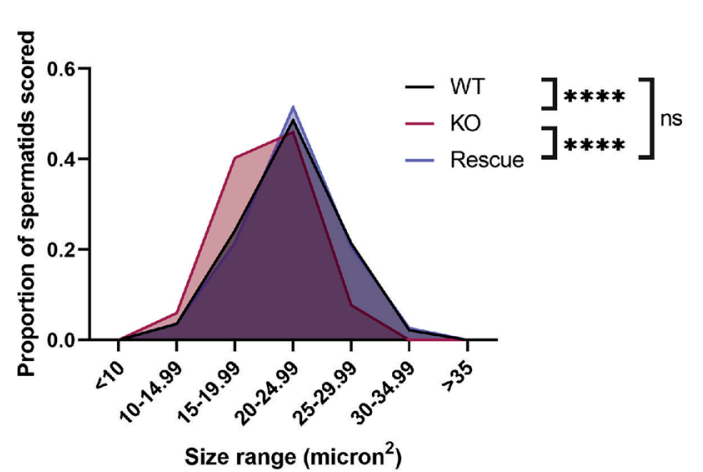

b

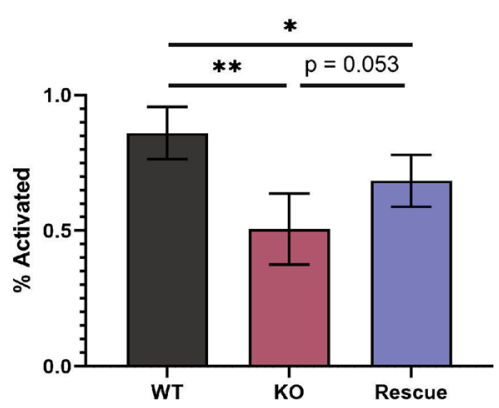

d
C

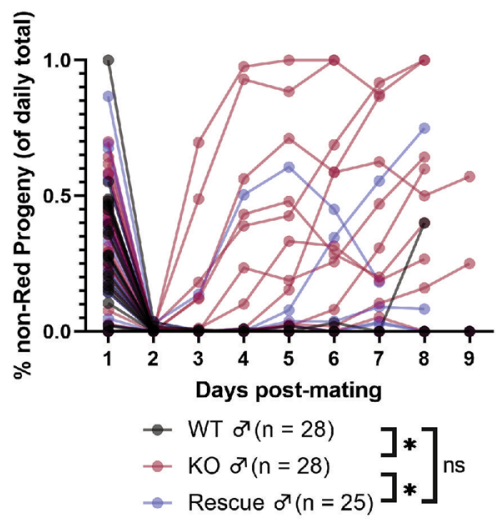

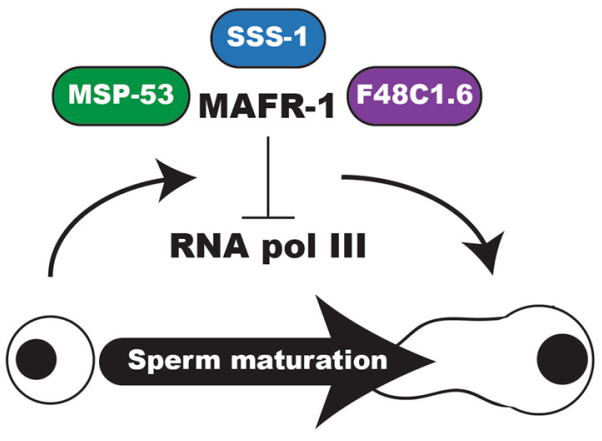

Figure 4. Germline expression of WT mafr-1 rescues sperm quality phenotypes. (a) Spermatid size in WT, mafr-1 (KO), and germline-specific rescue males. See Supplemental Data Set for further statistical analysis. (b) In vitro Pronase activation of WT, mafr-1 (KO), and germline-specific rescue male spermatids. mafr-1 $(\mathrm{KO}) /$ Rescue comparison done via unpaired, one-tailed t-test. Experiments done in biological triplicate. (c) Proportion of progeny fertilized by self-sperm from WT, mafr-1 (KO), and germline-specific rescue-mated hermaphrodites. WT and mafr-1 (KO) data are same data presented in Fig. 3e. (d) Model of effects of MAFR-1 on spermatid quality. MAFR-1 interacts with MSP-53, SSS-1, and F48C1.6, and its repression of RNA pol III is required for proper sperm maturation (Adobe Illustrator v24.3). Unless otherwise specified, statistical comparisons made by Student's t-test (two-tailed). ns = no significance, ${ }^{\star} \mathrm{p}<0.05,{ }^{* *} \mathrm{p}<0.01,{ }^{* * *} \mathrm{p}<0.001$, ${ }^{* * * *} \mathrm{p}<0.0001$.

Fig. S1c). This discrepancy could be explained by post-transcriptional regulatory pathways that govern intestinal synthesis of the vitellognenins, export from intestinal cells, or endocytosis in oocytes. Additionally, it is possible that mafr-1 RNAi results in different regulation of vitellogenins through persistent expression, as RNAi provides significant knockdown, but not complete ablation, of gene expression. RNAi is not uniformly effective across tissues, providing inefficient knockdown in the neurons ${ }^{51,52}$, pharyn $x^{53}$, and vulva ${ }^{49,61}$, which could be acting cell non-autonomously to cause pleiotropic gene expression phenotypes. As reproduction was generally normal in mafr-1 (KO) animals, it is not surprising that other regulatory mechanisms might restore homeostasis. Nevertheless, our studies in males, which do not express vitellogenins, identifies a novel role of mafr- 1 in proper sperm function.

Furthermore, it is likely that regulation of vitellogenin expression may be sensitive to diet, as mafr-1 expression and mafr-1-sensitive phenotypes are altered on an Escherichia coli K12/HT115 diet, in which the vast majority of RNAi studies are performed, relative to the standard E. coli B/OP50 diet ${ }^{8}$. Similarly, spermatid size appears to be sensitive to diet, with HT115-fed mafr-1 (KO) males producing significantly smaller sperm than those reared on OP50 (Fig. S3b-c). MAFR-1 plays an important role coordinating regulation of biosynthetic capacity in response to stimuli from the insulin signaling pathway $8,10,17,62,63$, which may be induced on the HT115 diet, as endogenous carbohydrates are 3- to 5-fold higher in HT115 relative to OP50 ${ }^{64}$.

Prior to this study, known physical interactors of MAF1 and its homologs were limited to regulatory kinases and phosphatases ${ }^{6-67}$, as well as RNA pol III subunits and transcription factors ${ }^{27-30}$. Although the molecular function of this interaction is not fully understood, the MSP family of proteins play multiple roles in sperm function and biogenesis ${ }^{32,33}$. Interaction of MAFR-1 with proteins expressed in a tissue-specific manner implies that MAFR-1 could play additional roles that are unique to distinct cell types. Furthermore, restoration of WT mafr- 1 expression in the germline partially rescued all measured metrics of sperm quality (Fig. 4a-c), indicating the cell-autonomous nature of the role of MAFR-1 in sperm maturation. To the best of our knowledge, this is the first instance of manipulation of any MAF1 homolog in any individual tissue of a multicellular organism. 
Taken together, our findings suggest that MAF1 may have unique roles in unique tissues that warrant further investigation.

While mafr-1 (KO) animals have similar lifespans (Fig. 1c) and developmental rates (Fig. 1b) to WT, germlinerescued mafr-1 (KO) animals take approximately two hours longer than WT or mafr-1 (KO) to become gravid adults (Fig. S3b). MAF1 is activated in human cells in response to serum starvation ${ }^{68,69}$, and confers starvation resistance in other organisms ${ }^{3,6}$. Expression of MAFR-1 under a non-endogenous promoter prevents it from being transcriptionally regulated by its normal cues, which could conceivably result in perceived starvation. In the germline, C. elegans have been shown to slow oogenesis in response to starvation ${ }^{70}$, and thus, pie-1 promoterdriven MAFR-1 expression may cause the observed delay in reproductive maturation of mafr-1 (KO) animals through perceived starvation in the germline.

MAF1 is generally viewed as a repressor of growth ${ }^{63}$. Seemingly contrary to this paradigm, spermatids from males lacking mafr-1 are smaller than those of WT males (Fig. 3a). In C. elegans, as sperm size is correlated with competitive advantage, and therefore general sperm quality ${ }^{24}$, small sperm can be an indication of poor sperm quality. RNA pol III transcripts make up an significant fraction of spermatid RNA populations ${ }^{71,72}$, and while mature spermatids are transcriptionally and translationally quiescent ${ }^{4,22}$, erroneous RNA pol III activity in germ cells likely results in lower quality, and therefore smaller, spermatids. Rescue of MAFR-1 expression in the germline rescued the diminished spermatid size of mafr-1 (KO) males, which suggests mafr-1 activity is required in a cell-specific manner for spermatid size (Fig. 4a). In addition, RNAi of the RNA pol III transcription factor $b r f-1$ restored spermatid size revealing that this phenotype is linked to aberrant RNA pol III activity (Fig. 3b). Similarly, sperm activation was rescued by restoration of WT MAFR-1 in the germline (Fig. 4b), but the effects of $b r f-1$ RNAi on activation were not significant. Based on the effect of $b r f-1$ RNAi in WT spermatids, it appears that the loss of RNA pol III activity is perhaps pleiotropic, potentially because of the role of proteostasis in sperm activation ${ }^{34,35}$. Nevertheless, the trend was toward restoring sperm activation defects stemming from loss of mafr- 1 when $b r f-1$ is reduced by RNAi (Fig. 3d). Our results suggest that tight regulation of RNA pol III activity, through MAFR-1, is important for proper sperm function.

In all instances we employed RNAi to explore the role of a gene in regulating sperm quality, we found significant effects on sperm size but not spermatid activation (Figs. 2c,d, 3b,d, Fig. S2d-f). While this suggests that sperm size is the more sensitive assay, these results do not necessarily indicate that the genes investigated do not play a role in sperm maturation. Male gametes appear to be another tissue in which RNAi efficiency is reduced $^{36,37}$, as several sperm-specific sterility phenotypes caused by genetic mutants cannot be recapitulated through $\mathrm{RNAi}^{37}$. Genetic manipulation is necessary to determine the role of these genes in spermiogenesis, but a lack of viable mutants leaves RNAi as the best currently available tool to study $s s s-1, F 48 C 1.6, m s p-53$, and $b r f$ 1 in this context. We note that our discovery of the sperm-specific phenotypes of mafr- 1 might not have been uncovered without the development of the CRISPR-generated null allele. In summary, our work defines three novel interactors of MAFR-1, and a unique role in the male germline affecting sperm maturation, likely through its regulation of RNA pol III activity (Fig. 4d).

\section{Experimental procedures}

Caenorhabditis elegans strains and maintenance. Caenorhabditis elegans were maintained at $20^{\circ} \mathrm{C}$ on $6 \mathrm{~cm}$ plates of Nematode Growth Medium (NGM) supplemented with streptomycin and seeded with OP50-1 E. coli. For RNAi experiments, NGM plates were supplemented with $5 \mathrm{mM}$ IPTG and $100 \mu \mathrm{g} / \mathrm{ml}$ carbenicillin and seeded with HT115 E. coli expressing dsRNA targeting gene as specified.

The following strains were used: wild type (WT) N2 Bristol, PHX557[mafr-1(syb557) I], SPC489 [mafr1(syb557) I; laxSi01-pie-1p::mafr-1::mafr-1 3'UTR cb-unc-119(+)II], WBM1143 [wbmIs67-eef-1A.1p::3XFL AG::wrmScarlet::unc-54 3'UTRV], SPC490 [mafr-1(syb557) I; wbmIs67-eft-3p::3XFLAG::wrmScarlet::unc-54 3'UTRV], SPC491 [mafr-1(syb557) I; laxSi01-pie-1p::mafr-1::mafr-1 3'UTR cb-unc-119(+)II; wbmIs67-eft3p::3XFLAG::wrmScarlet::unc-54 3UTRV], DH1033 [sqt-1(sc103) II; bIs1 - vit-2::GFP rol-6(su1006) X], SPC492 [mafr-1(syb557) I; sqt-1(sc103) II; bIs1 - vit-2::GFP rol-6(su1006) X].

Yeast-2-hybrid screen. Bait and prey plasmids were generated by cloning mafr-1 into pLexA and a C. elegans cDNA library into pACT2.2 (Addgene), respectively. Interaction was tested on synthetic complete agar that lacked leucine and tryptophan and was supplemented with $\mathrm{X}$ - $\alpha$-gal. Interactors were identified by transforming Y2HGold (Clontech) with bait (pLexA-MAFR-1) and the cDNA prey library. Positive clones were grown in the absence of tryptophan and sequenced. Sequenced clones were then retested individually.

Biochemical co-purification. MAFR-1 and respective interactors were cloned into MCS1 and MCS2 of pCOLA2, respectively. Origami K-12 E. coli were grown to stationary phase and expression of pCOLA2 vector was induced using IPTG for $2 \mathrm{~h}$. Cells were pelleted from $50 \mathrm{ml}$ induced culture, and frozen. Proteins were isolated and purified as in Ni-NTA Purification System protocol (Qiagen).

RNA extraction and gene expression. Worms were washed from plates using M9 containing $0.01 \%$ Triton-X100, washed twice in M9, and frozen at $-80{ }^{\circ} \mathrm{C}$ in $500 \mu \mathrm{l}$ TRI-Reagent (Zymo). Frozen worms were thawed on ice, and cuticles manually disrupted using $25 \mathrm{G}$ needle. RNA was extracted using Direct-zol RNA Miniprep kit (Zymo Research R2071). cDNA was synthesized using qScript reverse transcription kit (Quanta Biosciences), diluted, and quantitative PCR was performed using PerfecTA SYBR Green (Quanta Biosciences). All genes were normalized to expression of $s n b-1$. Primers used as previously described in Pradhan et al. and Khanna et al. ${ }^{16}$. New primers used in this study are as follows: 


\begin{tabular}{|l|l|l|}
\hline Gene name & Forward primer sequence & Reverse primer sequence \\
\hline$m s p-53$ & TGCCATAATCTTCACTGCGAG & $\begin{array}{l}\text { TCCTTTGGGTCGAGAACT } \\
\text { CC }\end{array}$ \\
\hline$s s s-1$ & CTACGGCATCACTTTTCGGG & $\begin{array}{l}\text { GAAACAGATTGAGCCGTG } \\
\text { CA }\end{array}$ \\
\hline$F 48 C 1.6$ & GGCGTAGTTCCTTTAGTGCG & $\begin{array}{l}\text { AGCTCGTGGAGATTGTTG } \\
\text { GA }\end{array}$ \\
\hline
\end{tabular}

Nile red lipid staining. Synchronous L4 animals were stained as $\mathrm{in}^{73}$. Briefly, animals were washed from plate, fixed in $40 \%$ isopropanol for $3 \mathrm{~min}$, stained in $0.03 \mathrm{mg} / \mathrm{ml} \mathrm{Nile} \mathrm{Red} \mathrm{in} 40 \%$ isopropanol for $2 \mathrm{~h}$, and destained in PBS with $0.01 \%$ Triton for $30 \mathrm{~min}$. Stained worms were mounted in de-staining solution and imaged using DIC and GFP filters on Zeiss Axio Imager, with ZEN software. Fluorescence of individual worms was measured using ImageJ software $(\mathrm{NIH})$.

Lifespan analysis. Lifespan data was collected as previously described ${ }^{40,74-76}$. In brief, plates containing synchronized populations were scored daily for survival, beginning at L4 stage. Animals were moved to new plates periodically during the reproductive span to remove progeny. Each line represents the average of two (in the case of WT) or three (in the case of mafr-1 (KO)) biological replicates of 50 animals each. Animals that died of bursting, matricide, or crawling off plate were censored.

Reproduction. Self-reproduction: L1-synchronized hermaphrodites were grown to L4 and single worms were placed on individual plates. Worms were transferred to fresh plates every $24 \mathrm{~h}$ until egg laying ceased, and progeny were counted $48 \mathrm{~h}$ after hermaphrodite was removed from plate.

Mated-reproduction: Single L4 hermaphrodites were placed with virgin day 1 adult males (harboring a CRISPR-integrated, single-copy, eef-1A.1 promoter-driven wrmScarlet transgene) in a 1:1 ratio on plates seeded with $20 \mu \mathrm{l}$ OP50, and allowed to mate overnight. Males were removed from plates, and hermaphrodites were transferred to fresh plates every $24 \mathrm{~h}$ until egg laying ceased. Progeny were counted and scored for wrmScarlet fluorescence $48 \mathrm{~h}$ after hermaphrodite was moved from plate. Animals were censored as "not sufficiently mated" if male sperm did not suppress self-progeny production to $<5 \%$ of the daily total on day 2 . Hermaphrodites were counted as producing non-Red progeny if $>2 \%$ of the total progeny produced after day 1 of adulthood were nonRed (progeny produced on the first day were not included due to variance in mating efficiency).

Sperm size analysis. Spermatids were isolated as previously described ${ }^{35}$. In brief, five virgin day 1 adult males were dissected in SM buffer containing dextrose to release spermatids. Spermatids were imaged using Zeiss Axio Imager, and size measured using ImageJ software. Unless otherwise indicated, unique WT controls were performed for each figure panel. For experiments in which sss-1, F48C1.6, and msp-53 RNAi were employed, animals were hatched on RNAi, and treated as OP50 animals-L4s were sequestered for $24 \mathrm{~h}$ and virgin day 1 adult males were dissected. Dissected gonads that released activated and mature sperm, instead of only spermatids were censored.

For experiments in which $b r f-1$ RNAi was employed, RNAi was administered post-developmentally: animals were hatched on OP50, and L4s were treated with RNAi and sequestered for $48 \mathrm{~h}$, then virgin day 2 adult males were dissected.

Spermatid activation assay. Five virgin males were dissected in SM buffer containing BSA ${ }^{35}$. An equal volume of SM buffer containing $400 \mu \mathrm{g} / \mathrm{ml}$ Pronase (Millipore Sigma) was added, and spermatids were allowed to activate for $15 \mathrm{~min}$. Images were manually scored, counting spermatids with pseudopodia as "activated." As with sperm size analysis, L4 males were sequestered for $24 \mathrm{~h}$ before dissection, except in the case of $b r f-1$ RNAi, in which L4 males were isolated for $48 \mathrm{~h}$.

VIT-2::GFP imaging/quantification. Synchronous animals were washed from plates and fixed in $40 \%$ isopropanol for $2 \mathrm{~h}$ before a 30-min wash in PBS with $0.01 \%$ Triton-X100. Fixed animals were mounted and imaged on a Zeiss Axio Imager, and fluorescence of most proximal oocyte was quantified using ImageJ software (NIH). In order to ensure animals of the same developmental stage were quantified, only animals with exactly two embryos in utero were imaged. Fluorescence intensity per animal is equal to the sum of the corrected total cell fluorescence (CTCF) of both proximal oocytes.

Developmental timing assay. Synchronous populations of L1 animals were dropped OP50, and beginning at $54 \mathrm{~h}$ post-drop scored for gravidity. Animals with uteruses containing at least one egg were considered to be gravid.

\section{Data availability}

All data are contained within the manuscript.

Received: 11 May 2020; Accepted: 27 October 2020

Published online: 09 November 2020 


\section{References}

1. Boguta, M., Czerska, K. \& Zoladek, T. Mutation in a new gene MAF1 affects tRNA suppressor efficiency in Saccharomyces cerevisiae. Gene 185, 291-296. https://doi.org/10.1016/s0378-1119(96)00669-5 (1997).

2. Pluta, K. et al. Maf1p, a negative effector of RNA polymerase III in Saccharomyces cerevisiae. Mol. Cell. Biol. 21, 5031-5040. https ://doi.org/10.1128/MCB.21.15.5031-5040.2001 (2001).

3. Upadhya, R., Lee, J. \& Willis, I. M. Maf1 is an essential mediator of diverse signals that repress RNA polymerase III transcription. Mol. Cell. 10, 1489-1494 (2002).

4. Johnson, S. S., Zhang, C., Fromm, J., Willis, I. M. \& Johnson, D. L. Mammalian Maf1 is a negative regulator of transcription by all three nuclear RNA polymerases. Mol. Cell. 26, 367-379. https://doi.org/10.1016/j.molcel.2007.03.021 (2007).

5. Soprano, A. S., Abe, V. Y., Smetana, J. H. \& Benedetti, C. E. Citrus MAF1, a repressor of RNA polymerase III, binds the Xanthomonas citri canker elicitor PthA4 and suppresses citrus canker development. Plant Physiol. 163, 232-242. https://doi. org/10.1104/pp.113.224642 (2013).

6. McLean, K. J. \& Jacobs-Lorena, M. Plasmodium falciparum Maf1 confers survival upon amino acid starvation. $m$ Bio https://doi. org/10.1128/mBio.02317-16 (2017).

7. Pernas, L. et al. Toxoplasma effector MAF1 mediates recruitment of host mitochondria and impacts the host response. PLoS Biol. 12, e1001845. https://doi.org/10.1371/journal.pbio.1001845 (2014).

8. Khanna, A., Johnson, D. L. \& Curran, S. P. Physiological roles for mafr-1 in reproduction and lipid homeostasis. Cell. Rep. 9, 2180-2191. https://doi.org/10.1016/j.celrep.2014.11.035 (2014).

9. Mierzejewska, J. \& Chreptowicz, K. Lack of Mafl enhances pyruvate kinase activity and fermentative metabolism while influencing lipid homeostasis in Saccharomyces cerevisiae. FEBS Lett. 590, 93-100. https://doi.org/10.1002/1873-3468.12033 (2016).

10. Palian, B. M. et al. Mafl is a novel target of PTEN and PI3K signaling that negatively regulates oncogenesis and lipid metabolism. PLoS Genet. 10, e1004789. https://doi.org/10.1371/journal.pgen.1004789 (2014).

11. Cai, Y. \& Wei, Y. H. Stress resistance and lifespan are increased in C. elegans but decreased in S. cerevisiae by mafr-1/maf1 deletion. Oncotarget 7, 10812-10826. https://doi.org/10.18632/oncotarget.7769 (2016).

12. Shetty, M. et al. Maf1-dependent transcriptional regulation of tRNAs prevents genomic instability and is associated with extended lifespan. Aging Cell 19, e13068. https://doi.org/10.1111/acel.13068 (2020).

13. Chen, C. Y. et al. Maf1 and repression of RNA polymerase III-mediated transcription drive adipocyte differentiation. Cell Rep. 24, 1852-1864. https://doi.org/10.1016/j.celrep.2018.07.046 (2018).

14. Rohira, A. D., Chen, C. Y., Allen, J. R. \& Johnson, D. L. Covalent small ubiquitin-like modifier (SUMO) modification of Maf1 protein controls RNA polymerase III-dependent transcription repression. J Biol Chem 288, 19288-19295. https://doi.org/10.1074/ jbc.M113.473744 (2013).

15. Bonhoure, N. et al. Loss of the RNA polymerase III repressor MAF1 confers obesity resistance. Genes Dev. 29, 934-947. https:// doi.org/10.1101/gad.258350.115 (2015).

16. Pradhan, A., Hammerquist, A. M., Khanna, A. \& Curran, S. P. The C-box region of MAF1 regulates transcriptional activity and protein stability. J. Mol. Biol. 429, 192-207. https://doi.org/10.1016/j.jmb.2016.12.012 (2017).

17. Rideout, E. J., Marshall, L. \& Grewal, S. S. Drosophila RNA polymerase III repressor Mafl controls body size and developmental timing by modulating tRNAiMet synthesis and systemic insulin signaling. Proc. Natl. Acad. Sci. U.S.A. 109, 1139-1144. https:// doi.org/10.1073/pnas.1113311109 (2012).

18. Marygold, S. J. et al. The ribosomal protein genes and Minute loci of Drosophila melanogaster. Genome Biol. 8, R216. https://doi. org/10.1186/gb-2007-8-10-r216 (2007).

19. Alla, R. K. \& Cairns, B. R. RNA polymerase III transcriptomes in human embryonic stem cells and induced pluripotent stem cells, and relationships with pluripotency transcription factors. PLoS ONE 9, e85648. https://doi.org/10.1371/journal.pone.00856 48 (2014).

20. Nakamoto, T. et al. Impaired spermatogenesis and male fertility defects in CIZ/Nmp4-disrupted mice. Genes Cells 9, 575-589. https://doi.org/10.1111/j.1356-9597.2004.00746.x (2004).

21. Johnson, G. D. et al. Cleavage of rRNA ensures translational cessation in sperm at fertilization. Mol. Hum. Reprod. 17, 721-726. https://doi.org/10.1093/molehr/gar054 (2011).

22. Kierszenbaum, A. L. \& Tres, L. L. Structural and transcriptional features of the mouse spermatid genome. J. Cell. Biol. 65, 258-270. https://doi.org/10.1083/jcb.65.2.258 (1975).

23. Gomendio, M. \& Roldan, E. R. Sperm competition influences sperm size in mammals. Proc. Biol. Sci. 243, 181-185. https://doi. org/10.1098/rspb.1991.0029 (1991).

24. LaMunyon, C. W. \& Ward, S. Larger sperm outcompete smaller sperm in the nematode Caenorhabditis elegans. Proc. Biol. Sci. 265, 1997-2002. https://doi.org/10.1098/rspb.1998.0531 (1998).

25. LaMunyon, C. W. \& Ward, S. Evolution of sperm size in nematodes: Sperm competition favours larger sperm. Proc. Biol. Sci. 266, 263-267. https://doi.org/10.1098/rspb.1999.0631 (1999).

26. LaMunyon, C. W. \& Ward, S. Sperm precedence in a hermaphroditic nematode (Caenorhabditis elegans) is due to competitive superiority of male sperm. Experientia 51, 817-823 (1995).

27. Desai, N. et al. Two steps in Mafl-dependent repression of transcription by RNA polymerase III. J. Biol. Chem. 280, 6455-6462. https://doi.org/10.1074/jbc.M412375200 (2005).

28. Lee, Y. L. et al. MAF1 represses CDKN1A through a Pol III-dependent mechanism. Elife 4, e06283. https://doi.org/10.7554/eLife $06283(2015)$

29. Oficjalska-Pham, D. et al. General repression of RNA polymerase III transcription is triggered by protein phosphatase type 2A-mediated dephosphorylation of Maf1. Mol. Cell. 22, 623-632. https://doi.org/10.1016/j.molcel.2006.04.008 (2006).

30. Reina, J. H., Azzouz, T. N. \& Hernandez, N. Maf1, a new player in the regulation of human RNA polymerase III transcription. PLOS ONE 1, e134. https://doi.org/10.1371/journal.pone.0000134 (2006).

31. Yook, K. et al. WormBase 2012: More genomes, more data, new website. Nucleic Acids Res. 40, D735-741. https://doi.org/10.1093/ nar/gkr954 (2012).

32. Smith, H. E. Nematode sperm motility. WormBook https://doi.org/10.1895/wormbook.1.68.2 (2014).

33. Smith, H. E. \& Ward, S. Identification of protein-protein interactions of the major sperm protein (MSP) of Caenorhabditis elegans. J. Mol. Biol. 279, 605-619. https://doi.org/10.1006/jmbi.1998.1793 (1998).

34. Ward, S., Hogan, E. \& Nelson, G. A. The initiation of spermiogenesis in the nematode Caenorhabditis elegans. Dev. Biol. 98, 70-79 (1983).

35. Yen, C. A., Ruter, D. L., Turner, C. D., Pang, S. \& Curran, S. P. Loss of flavin adenine dinucleotide (FAD) impairs sperm function and male reproductive advantage in C. elegans. Elife https://doi.org/10.7554/eLife.52899 (2020).

36. Liau, W. S., Nasri, U., Elmatari, D., Rothman, J. \& LaMunyon, C. W. Premature sperm activation and defective spermatogenesis caused by loss of spe-46 function in Caenorhabditis elegans. PLoS ONE 8, e57266. https://doi.org/10.1371/journal.pone.0057266 (2013).

37. del Castillo-Olivares, A., Kulkarni, M. \& Smith, H. E. Regulation of sperm gene expression by the GATA factor ELT-1. Dev. Biol. 333, 397-408. https://doi.org/10.1016/j.ydbio.2009.06.044 (2009). 
38. Yen, C. A. \& Curran, S. P. Gene-diet interactions and aging in C. elegans. Exp. Gerontol. 86, 106-112. https://doi.org/10.1016/j. exger.2016.02.012 (2016).

39. Lynn, D. A. et al. Omega-3 and -6 fatty acids allocate somatic and germline lipids to ensure fitness during nutrient and oxidative stress in Caenorhabditis elegans. Proc. Natl. Acad. Sci. U.S.A. 112, 15378-15383. https://doi.org/10.1073/pnas.1514012112 (2015).

40. Pang, S. \& Curran, S. P. Adaptive capacity to bacterial diet modulates aging in C. elegans. Cell. Metab. 19, 221-231. https://doi. org/10.1016/j.cmet.2013.12.005 (2014).

41. Pang, S., Lynn, D. A., Lo, J. Y., Paek, J. \& Curran, S. P. SKN-1 and Nrf2 couples proline catabolism with lipid metabolism during nutrient deprivation. Nat. Commun. 5, 5048. https://doi.org/10.1038/ncomms6048 (2014).

42. Nhan, J. D. et al. Redirection of SKN-1 abates the negative metabolic outcomes of a perceived pathogen infection. Proc. Natl. Acad. Sci. U.S.A. 116, 22322-22330. https://doi.org/10.1073/pnas.1909666116 (2019).

43. Khanna, A., Pradhan, A. \& Curran, S. P. Emerging roles for Maf1 beyond the regulation of RNA polymerase III activity. J. Mol. Biol. 427, 2577-2585. https://doi.org/10.1016/j.jmb.2015.06.022 (2015).

44. Buratowski, S. \& Zhou, H. A suppressor of TBP mutations encodes an RNA polymerase III transcription factor with homology to TFIIB. Cell 71, 221-230. https://doi.org/10.1016/0092-8674(92)90351-c (1992).

45. Colbert, T. \& Hahn, S. A yeast TFIIB-related factor involved in RNA polymerase III transcription. Genes Dev. 6, 1940-1949. https ://doi.org/10.1101/gad.6.10.1940 (1992).

46. Kassavetis, G. A. et al. The role of the TATA-binding protein in the assembly and function of the multisubunit yeast RNA polymerase III transcription factor. TFIIIB. Cell 71, 1055-1064. https://doi.org/10.1016/0092-8674(92)90399-w (1992).

47. Larminie, C. G. \& White, R. J. Identification of a putative BRF homologue in the genome of Caenorhabditis elegans. DNA Seq. 9, 49-58. https://doi.org/10.3109/10425179809050025 (1998).

48. Lopez-De-Leon, A., Librizzi, M., Puglia, K. \& Willis, I. M. PCF4 encodes an RNA polymerase III transcription factor with homology to TFIIB. Cell 71, 211-220. https://doi.org/10.1016/0092-8674(92)90350-1 (1992).

49. Conte, D., MacNeil, L. T., Walhout, A. J. M. \& Mello, C. C. RNA Interference in Caenorhabditis elegans. Curr .Protoc. Mol. Biol. 109, 262321-262330. https://doi.org/10.1002/0471142727.mb2603s109 (2015).

50. Fire, A. Z. Gene silencing by double-stranded RNA. Cell Death Differ. 14, 1998-2012. https://doi.org/10.1038/sj.cdd.4402253 (2007).

51. Kamath, R. S., Martinez-Campos, M., Zipperlen, P., Fraser, A. G. \& Ahringer, J. Effectiveness of specific RNA-mediated interference through ingested double-stranded RNA in Caenorhabditis elegans. Genome Biol. https://doi.org/10.1186/gb-2000-2-1-resea rch0002 (2001).

52. Timmons, L., Court, D. L. \& Fire, A. Ingestion of bacterially expressed dsRNAs can produce specific and potent genetic interference in Caenorhabditis elegans. Gene 263, 103-112 (2001).

53. Shiu, P. K. \& Hunter, C. P. Early developmental exposure to dsRNA is critical for initiating efficient nuclear RNAi in C. elegans. Cell. Rep. 18, 2969-2978. https://doi.org/10.1016/j.celrep.2017.03.002 (2017).

54. Merritt, C., Rasoloson, D., Ko, D. \& Seydoux, G. 3'UTRs are the primary regulators of gene expression in the C. elegans germline. Curr. Biol. 18, 1476-1482. https://doi.org/10.1016/j.cub.2008.08.013 (2008).

55. Merritt, C. \& Seydoux, G. Transgenic solutions for the germline. WormBook https://doi.org/10.1895/wormbook.1.148.1 (2010).

56. Tenenhaus, C., Schubert, C. \& Seydoux, G. Genetic requirements for PIE-1 localization and inhibition of gene expression in the embryonic germ lineage of Caenorhabditis elegans. Dev. Biol. 200, 212-224. https://doi.org/10.1006/dbio.1998.8940 (1998).

57. Kimble, J. E. Strategies for control of pattern formation in Caenorhabditis elegans. Philos. Trans. R. Soc. Lond. B Biol. Sci. 295, 539-551. https://doi.org/10.1098/rstb.1981.0159 (1981).

58. Ebbing, A. et al. Spatial transcriptomics of $C$. elegans males and hermaphrodites identifies sex-specific differences in gene expression patterns. Dev. Cell. 47, 801-813. https://doi.org/10.1016/j.devcel.2018.10.016 (2018).

59. Grun, D. et al. Conservation of mRNA and protein expression during development of C. elegans. Cell. Rep. 6, 565-577. https:// doi.org/10.1016/j.celrep.2014.01.001 (2014).

60. Han, Y. N. et al. PIWI proteins and PIWI-interacting RNA: Emerging roles in cancer. Cell. Physiol. Biochem. 44, 1-20. https://doi. org/10.1159/000484541 (2017).

61. Parrish, S., Fleenor, J., Xu, S., Mello, C. \& Fire, A. Functional anatomy of a dsRNA trigger: Differential requirement for the two trigger strands in RNA interference. Mol. Cell. 6, 1077-1087. https://doi.org/10.1016/s1097-2765(00)00106-4 (2000).

62. Marshall, L., Rideout, E. J. \& Grewal, S. S. Nutrient/TOR-dependent regulation of RNA polymerase III controls tissue and organismal growth in Drosophila. Embo. J. 31, 1916-1930. https://doi.org/10.1038/emboj.2012.33 (2012).

63. Li, Y. et al. MAF1 suppresses AKT-mTOR signaling and liver cancer through activation of PTEN transcription. Hepatology 63, 1928-1942. https://doi.org/10.1002/hep.28507 (2016).

64. Brooks, K. K., Liang, B. \& Watts, J. L. The influence of bacterial diet on fat storage in C. elegans. PLoS ONE 4, e7545. https://doi. org/10.1371/journal.pone.0007545 (2009).

65. Kantidakis, T., Ramsbottom, B. A., Birch, J. L., Dowding, S. N. \& White, R. J. mTOR associates with TFIIIC, is found at tRNA and 5S rRNA genes, and targets their repressor Maf1. Proc. Natl. Acad. Sci. U.S.A. 107, 11823-11828. https://doi.org/10.1073/pnas.10051 88107 (2010).

66. Michels, A. A. et al. mTORC1 directly phosphorylates and regulates human MAF1. Mol. Cell. Biol. 30, 3749-3757. https://doi. org/10.1128/MCB.00319-10 (2010).

67. Shor, B. et al. Requirement of the mTOR kinase for the regulation of Maf1 phosphorylation and control of RNA polymerase IIIdependent transcription in cancer cells. J. Biol. Chem. 285, 15380-15392. https://doi.org/10.1074/jbc.M109.071639 (2010).

68. Goodfellow, S. J. et al. Regulation of RNA polymerase III transcription by Maf1 in mammalian cells. J. Mol. Biol. 378, 481-491. https://doi.org/10.1016/j.jmb.2008.02.060 (2008).

69. Orioli, A., Praz, V., Lhote, P. \& Hernandez, N. Human MAF1 targets and represses active RNA polymerase III genes by preventing recruitment rather than inducing long-term transcriptional arrest. Genome Res. 26, 624-635. https://doi.org/10.1101/gr.20140 $0.115(2016)$.

70. Seidel, H. S. \& Kimble, J. The oogenic germline starvation response in C. elegans. PLoS ONE 6, e28074. https://doi.org/10.1371/ journal.pone.0028074 (2011).

71. Georgiadis, A. P. et al. High quality RNA in semen and sperm: Isolation, analysis and potential application in clinical testing. J. Urol. 193, 352-359. https://doi.org/10.1016/j.juro.2014.07.107 (2015).

72. Ma, X. et al. Characterisation of Caenorhabditis elegans sperm transcriptome and proteome. BMC Genom. 15, 168. https://doi. org/10.1186/1471-2164-15-168 (2014).

73. Escorcia, W., Ruter, D. L., Nhan, J. \& Curran, S. P. Quantification of lipid abundance and evaluation of lipid distribution in Caenorhabditis elegans by nile red and oil red O staining. J. Vis. Exp. https://doi.org/10.3791/57352 (2018).

74. Dalton, H. M. \& Curran, S. P. Hypodermal responses to protein synthesis inhibition induce systemic developmental arrest and AMPK-dependent survival in Caenorhabditis elegans. PLoS Genet. 14, e1007520. https://doi.org/10.1371/journal.pgen.1007520 (2018).

75. Haghani, A. et al. Air pollution alters Caenorhabditis elegans development and lifespan: Responses to traffic-related nanoparticulate matter (nPM). J. Gerontol. A Biol. Sci. Med. Sci. https://doi.org/10.1093/gerona/glz063 (2019).

76. Paek, J. et al. Mitochondrial SKN-1/Nrf mediates a conserved starvation response. Cell. Metab. 16, 526-537. https://doi. org/10.1016/j.cmet.2012.09.007 (2012). 


\title{
Acknowledgements
}

We thank J. Gonzalez and L. Thomas for technical assistance; C-A. Yen for critical reading of the manuscript. Some strains were provided by the CGC, which is funded by the NIH Office of Research Infrastructure Programs (P40 OD010440).

\section{Author contributions}

S.P.C. designed the study; A.M.H. performed the experiments; A.M.H. and S.P.C. analyzed data. A.M.H. and S.P.C. wrote and revised the manuscript.

\section{Funding}

This work was funded by the NIH R01GM109028 and R01AG058610 to S.P.C. and T32AG000037 to A.MH. The content is solely the responsibility of the authors and does not necessarily represent the official views of the National Institutes of Health.

\section{Competing interests}

The authors declare no competing interests.

\section{Additional information}

Supplementary information is available for this paper at https://doi.org/10.1038/s41598-020-76423-5.

Correspondence and requests for materials should be addressed to S.P.C.

Reprints and permissions information is available at www.nature.com/reprints.

Publisher's note Springer Nature remains neutral with regard to jurisdictional claims in published maps and institutional affiliations.

\begin{abstract}
(c) (1) Open Access This article is licensed under a Creative Commons Attribution 4.0 International License, which permits use, sharing, adaptation, distribution and reproduction in any medium or format, as long as you give appropriate credit to the original author(s) and the source, provide a link to the Creative Commons licence, and indicate if changes were made. The images or other third party material in this article are included in the article's Creative Commons licence, unless indicated otherwise in a credit line to the material. If material is not included in the article's Creative Commons licence and your intended use is not permitted by statutory regulation or exceeds the permitted use, you will need to obtain permission directly from the copyright holder. To view a copy of this licence, visit http://creativecommons.org/licenses/by/4.0/.
\end{abstract}

(c) The Author(s) 2020 